\title{
N-Methyl-2,6-di-tert-butyl-4,4'-pyrylogen. A dialkyl example of a new class of electron transfer sensitizers
}

\author{
Ajaya Kumar Sankara Warrier, and Edward L. Clennan* \\ Department of Chemistry, University of Wyoming, 1000 East University Avenue, Laramie WY \\ 82071 \\ E-mail: clennane@uwyo.edu
}

\section{Dedicated to Prof. Nicolò Vivona on the occasion of his $70^{\text {th }}$ birthday}

\begin{abstract}
The synthesis of the first dialkyl substituted pyrylogen, $N$-methyl-2,6-di-tert-butyl-4,4'pyrylogen bis-tetrafluoroborate is reported. The electrochemical and photophysical behavior of this new pyrylogen is compared to the previously reported diphenyl-substituted analogue, $N$ methyl-2,6-diphenyl-4,4'-pyrylogen. Insight into the structures and properties of this new pyrylogen and its radical cation and neutral redox partners is also provided using the B3LYP/6$31 \mathrm{G}(\mathrm{d})$ computational model.
\end{abstract}

Keywords: Pyrylium salts, viologens, fluorescence, phosphorescence, cyclic voltammetry, laser flash photolysis

\section{Introduction}

The explosion of interest in "photoinduced electron transfer" (PET) reactions can be readily demonstrated by searching for this term in the SciFinder Scholar database. ${ }^{1}$ The one manuscript located with this term published in the 1950s increases to 3 in the $1960 \mathrm{~s}, 47$ in the $1970 \mathrm{~s}, 570$ in the 1980 s, 3254 in the 1990 s, and 3582 since 2000. The remarkable interest in this topic reflects both the importance of this process in biological systems and the utility of PET to make new materials. Unfortunately, the low quantum yields of many photoinduced electron transfers provide a significant impediment to achieving an energy efficient environmentally green process. These low efficiencies can be attributed in many cases to energy wasting return electron transfer (RET) in the ion pair intermediates.

The problem of low quantum yields has been addressed over the years with several clever approaches. These approaches include: (1) the generation of triplet ion pair intermediates in which RET is spin-forbidden; ${ }^{2}$ (2) the use of co-sensitizers; ${ }^{3}$ (3) construction of electron transfer 
chain reactions in which a single electron transfer to the sensitizer is amplified by the propagation steps, ${ }^{4}$ (4) thermodynamic manipulations of the energetics of RET to put the system deep in the Marcus inverted region where RET is too slow to compete with the chemical reaction of interest; ${ }^{5,6}(5)$ the use of steric effects in either the substrate or sensitizer to increase substratesensitizer distance in the ion pair intermediate decreasing the electronic coupling matrix for RET; $^{7}$ and (6) the design of charge shift reactions (Eqn. 1 in Scheme 1) which eliminate coulombic attraction between the donor and acceptor and enhances diffusive separation at the expense of RET. ${ }^{6,8}$

$$
\begin{aligned}
& {\left[\operatorname{Sen}^{\oplus}\right] \stackrel{\text { hv }}{\longrightarrow}\left[\operatorname{Sen}^{\oplus}\right] \stackrel{\text { Substrate }}{\longrightarrow} \underbrace{\left[\text { Sen Substrate. }^{\oplus}\right]}_{\begin{array}{c}
\text { no coulombic } \\
\text { attraction }
\end{array}} \stackrel{\text { fast }}{\longrightarrow} \text { Sen }+ \text { Substrate. }} \\
& \text { "Free ion" }
\end{aligned}
$$

Scheme 1

Recently we reported the synthesis of 2,6-diaryl-pyrylogens, $\mathbf{1}^{2+}$, a new class of electron transfer sensitizers, that are hybrids of pyrylium cations, $\mathbf{2}^{+}$, and viologen dications, $\mathbf{3}^{\mathbf{2 +}} \cdot{ }^{2}$ These dications were designed to enhance diffusive separation as a result of repulsive coulombic interaction in the charge shift product (Eqn 2; Scheme 1) and consequently further suppress RET.
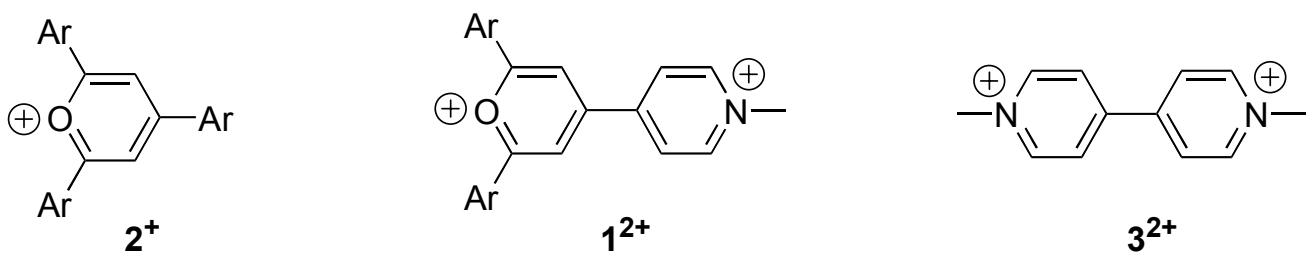

These new sensitizers show sufficient solubility in acetonitrile, reduced solubility in methylene chloride, and are too insoluble in less polar solvents to function as electron transfer sensitizers. The lack of solubility over a wide range of solvent polarities is a concern since it has been demonstrated that quantum yields of ion pair separation can increase as the solvent polarity is decreased. ${ }^{10}$ This counter intuitive observation has been attributed to a decrease in the solvent reorganization energy in the less polar solvents. ${ }^{11}$ In the Marcus inverted region (i.e. $-\Delta G^{o}$ RET $>>>$; Eqn. 3) ${ }^{12,13}$ a decrease in the solvent reorganization energy (i.e. decreasing solvent polarity) and the concomitant increase in driving force should lead to a decrease in the rate of 
RET and consequently an increase in the quantum yield of ion pair separation. The exact opposite results would be anticipated in the Marcus normal region (i.e. $-\Delta \mathrm{G}_{\mathrm{RET}}^{\mathrm{O}}<<<\lambda$; Eqn. 3) and a decrease in the solvent reorganization energy (i.e. decreasing solvent polarity) should lead to an increase in the rate of RET and concomitantly a decrease in the quantum yield of ion pair separation.

$$
k_{R E T}=\left(\frac{k T}{h}\right) \exp \left[-\left(\frac{\lambda}{4}\right) \frac{\left(1+\frac{\Delta G_{R E T}^{o}}{\lambda}\right)^{2}}{k T}\right\rfloor
$$

In this manuscript we describe the synthesis, electrochemical, and photophysical properties of $\mathrm{N}$-methyl-2,6-di-tert-butyl-4,4'-pyrylogen bistetrafluoroborate, $\mathbf{4}^{\mathbf{2}}$, that we envisioned would have better solubility over a range of solvents than the previously reported diaryl analogues.

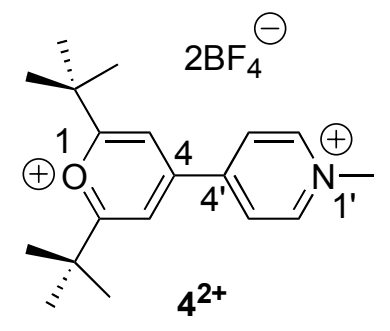

\section{Results and Discussion}

\section{Synthesis}

The synthesis of $\mathbf{4}^{\mathbf{2}}$ is outlined in Scheme 2 and follows for the most part the synthetic strategy designed for the diaryl pyrylogens. ${ }^{9}$ In the first step dimsyl anion is used to generate pinacolone enolate which adds twice to pyridine 4-carboxaldehyde presumably via an aldol condensation, loss of water, and Michael addition of the second equivalent to give the $\delta$-diketone, $\mathbf{5}$. This is followed by alkylation with Meerwin's salt to give the pyridinium salt $\mathbf{6}^{+}$. Finally a dehydrative cyclization followed by oxidation using trityl cation as a hydride acceptor generates the new dialkyl pyrylogen $4^{2+}$. 


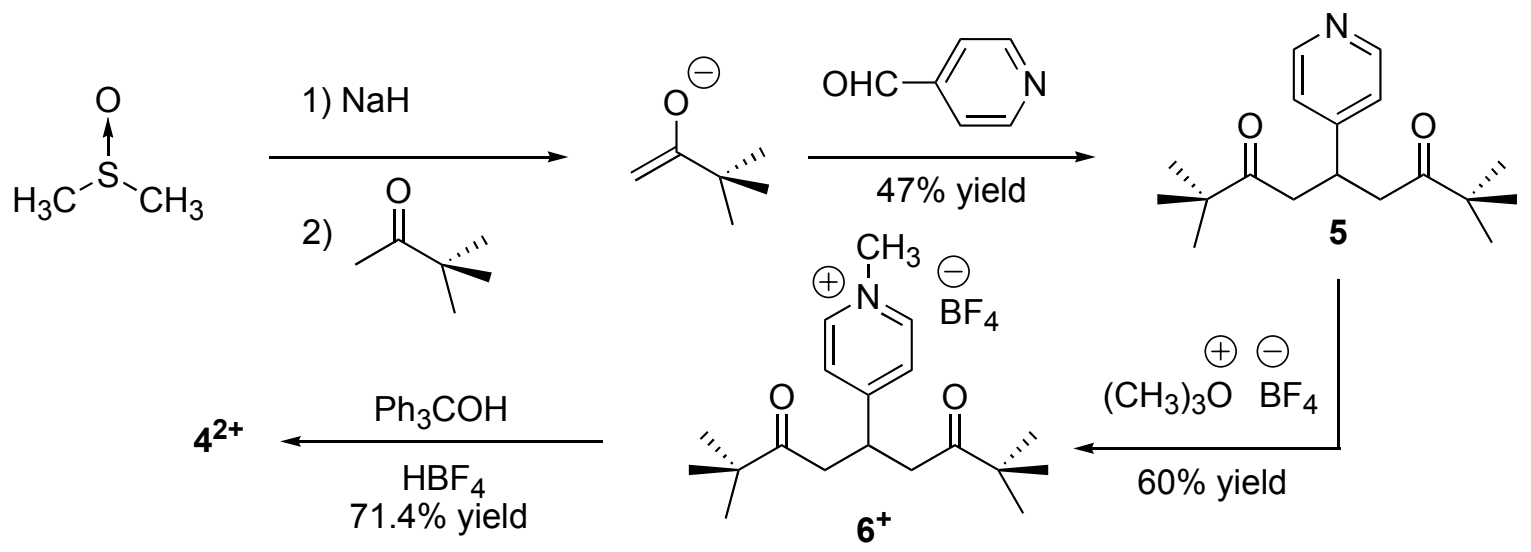

\section{Scheme 2}

The proton and carbon NMR data for $4^{2+}$ is very similar to that observed for $N$-methyl-2,6diphenyl-4,4'-pyrylogen bistetrafluoroborate, $\mathbf{1}^{\mathbf{2}}(\mathrm{Ar}=\mathrm{Ph})$. The hydrogen on the 3-position of the pyrylium ring $(8.28 \mathrm{ppm})$, however, is upfield of the corresponding hydrogen in $\mathbf{1}^{2+}(\mathrm{Ar}=\mathrm{Ph})$ at $8.80 \mathrm{ppm}$ and in 2,4,6-triphenyl pyrylium tetrafluoroborate, $\mathbf{2}^{+}(\mathrm{Ar}=\mathrm{Ph})$ at $8.70 \mathrm{ppm}$. On the other hand, the chemical shift of carbon-2,6 (190.7 ppm) bearing the tert-butyl group is substantially downfield of the corresponding carbons in $\mathbf{1}^{\mathbf{2}}(\mathrm{Ar}=\mathrm{Ph})$ and $\mathbf{2}^{+}(\mathrm{Ar}=\mathrm{Ph})$ at 174 and 170.1 ppm, respectively. 


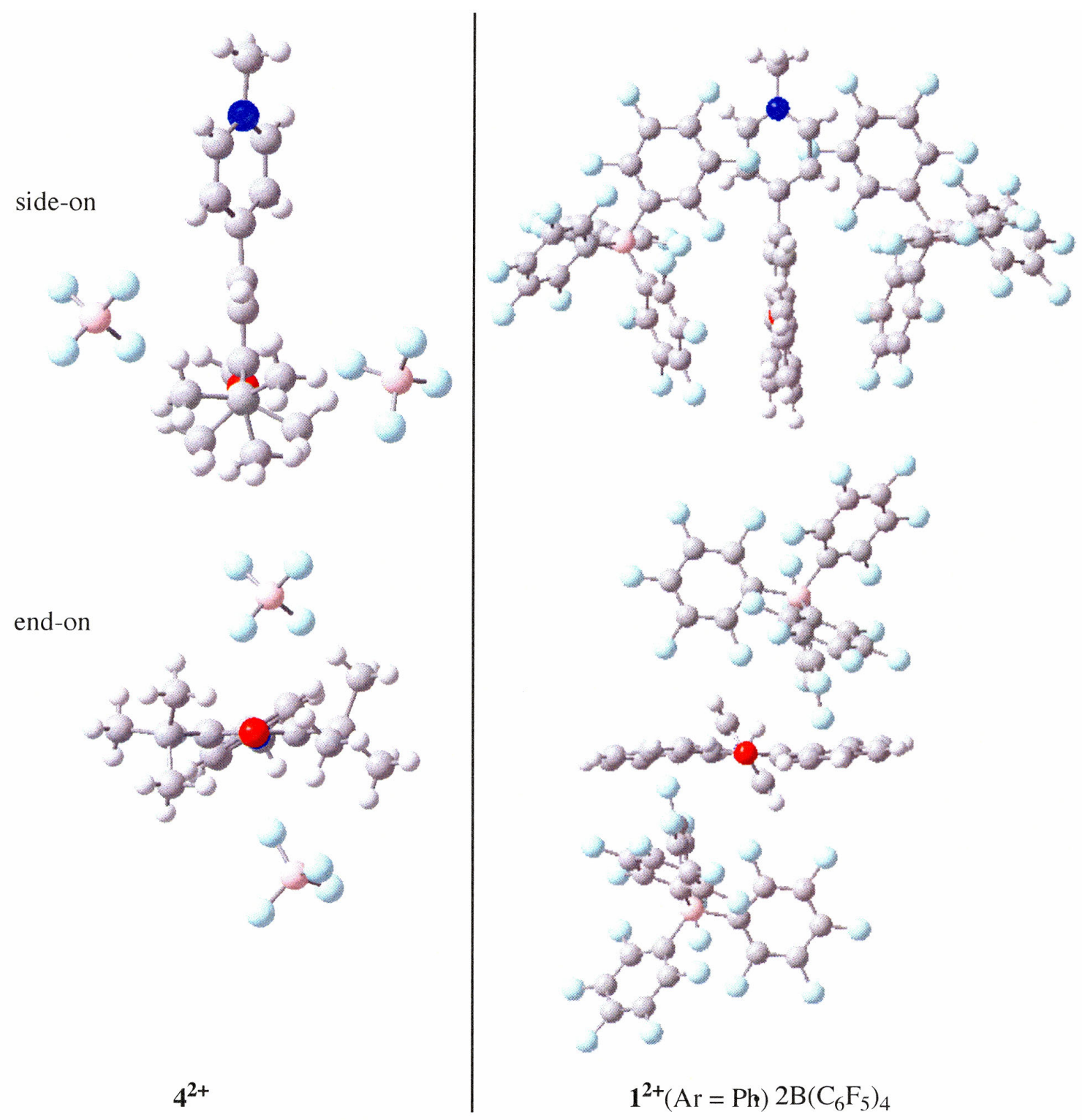

Figure 1. Side-on and End-on views of the crystal structures of $\mathbf{4}^{2+}$ and $\mathbf{1}^{2+}$

Conclusive evidence for the structure of $4^{2+}$ was obtained by X-Ray crystallography as shown in Figure 1. In this figure both side-on and end-on perspectives are compared to the same perspectives in $\mathbf{1}^{2+}(\mathrm{Ar}=\mathrm{Ph}) \bullet 2 \mathrm{~B}\left(\mathrm{C}_{6} \mathrm{~F}_{5}\right)_{4}$. Both structures are characterized by a significant twist from co-planarity around the bond connecting the pyrylium and pyridinium rings. This dihedral angle is larger in the diphenyl pyrylogen $\left(53.23^{\circ}\right)$ than in the di-tert-butyl pyrylogen $\left(29.99^{\circ}\right)$. The larger twist angle enhances the overlap in a laterally displaced perfluorophenyl-pyrydiniumperfluorophenyl cofacial sub-structure and leads to a favorable increase in the electrostatic/charge-transfer interaction. This cofacial arrangement is best viewed in the end-on perspective of $\mathbf{1}^{2+}(\mathrm{Ar}=\mathrm{Ph}) \bullet 2 \mathrm{~B}\left(\mathrm{C}_{6} \mathrm{~F}_{5}\right)_{4}$ depicted in the lower right hand corner of Figure 1. The 
pyrylogen cores are also sandwiched between the two counterions. In $\mathbf{1}^{2+}(\mathrm{Ar}=\mathrm{Ph}) \bullet 2 \mathrm{~B}\left(\mathrm{C}_{6} \mathrm{~F}_{5}\right)_{4}$ these counterions are symmetrically disposed but in $4^{2+}$ they adopt crystallographically distinct sites. In both cases the anions are more closely associated with the pyrylium rather than with the pyridinium ring. This is most clearly evident in the perspective of $\mathbf{4}^{2+}$ shown in the upper left hand corner of Figure 1. The differences in the $\mathrm{B}-\mathrm{O}_{\text {pyrylium }}$ and $\mathrm{B}-\mathrm{N}_{\text {pyridinium }}$ distances in $4^{2+}$ (B$\mathrm{O} / \mathrm{B}-\mathrm{N}=4.19 \AA / 6.39 \AA$ and $4.08 \AA / 7.84 \AA)$ are much larger than that observed in $\mathbf{1}^{2+}(\mathrm{Ar}=$ $\mathrm{Ph}) \bullet 2 \mathrm{~B}\left(\mathrm{C}_{6} \mathrm{~F}_{5}\right)_{4}(\mathrm{~B}-\mathrm{O} / \mathrm{B}-\mathrm{N}=6.29 \AA / 7.15 \AA)$. We suggest that the location of the counterions reflect the greater amount of positive charge that resides on the pyrylium in comparison to the pyridinium ring. This suggestion is supported by both Mulliken and NPA population analyses on the B3LYP/6-31g(d) minimized structure of $4^{2+}$.

\section{Electrochemistry}

Pyrylogens $\mathbf{1}^{2+}(\mathrm{Ar}=\mathrm{Ph}) \bullet 2 \mathrm{BF}_{4}$ and $\mathbf{4}^{2+}$ both exhibit, even at scan rates of $50 \mathrm{mV} / \mathrm{sec}$ on glassy carbon, two quasireversible cyclic voltammetry peaks (Figure. 2) consistent with formation of stable radical cation and neutral redox partners. (Eqn. 4) The first and second reduction potentials of pyrylogen $\mathbf{1}^{2+}(\mathrm{Ar}=\mathrm{Ph}) \bullet 2 \mathrm{BF}_{4}$ at $+0.17 \mathrm{~V}$ and $-0.35 \mathrm{~V}$ vs SCE are approximately $100 \mathrm{mV}$ anodic of the corresponding reductions of $4^{2+}$ at $+0.07 \mathrm{~V}$ and $-0.44 \mathrm{~V}$ vs SCE, respectively. We attribute the easier reductions of $\mathbf{1}^{2+}(\mathrm{Ar}=\mathrm{Ph}) \cdot 2 \mathrm{BF}_{4}$ to the increased electron withdrawing ability of $\mathrm{Ph}\left(\sigma_{\mathrm{p}}=+0.05\right)$ in comparison to the tert-butyl $\left(\sigma_{\mathrm{p}}=-0.15\right)$ substituent. This substituent effect is small, however, in comparison to the $520 \mathrm{mV}$ increase in the ease of reduction realized by replacing the 4-phenyl ring in $2^{+}(\mathrm{Ar}=\mathrm{Ph})$ with a $\mathrm{N}$-methyl-pyridinium ring to give $\mathbf{1}^{2+}(\mathrm{Ar}=\mathrm{Ph}) \cdot 2 \mathrm{BF}_{4}$.
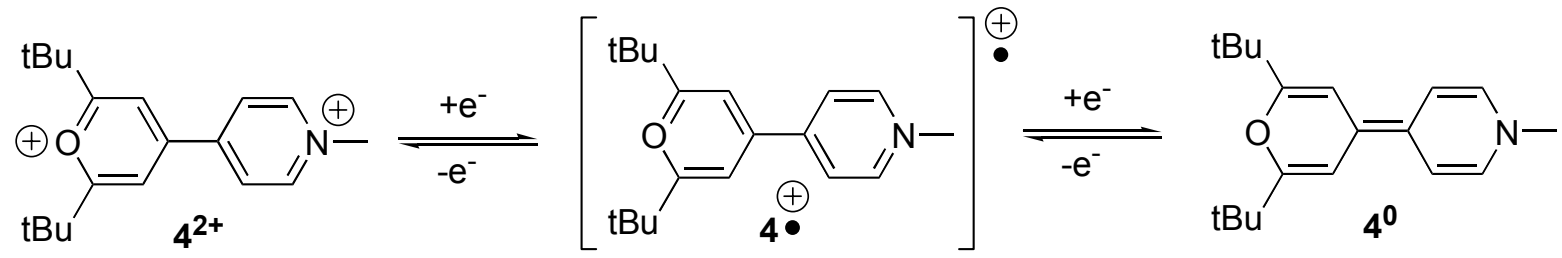


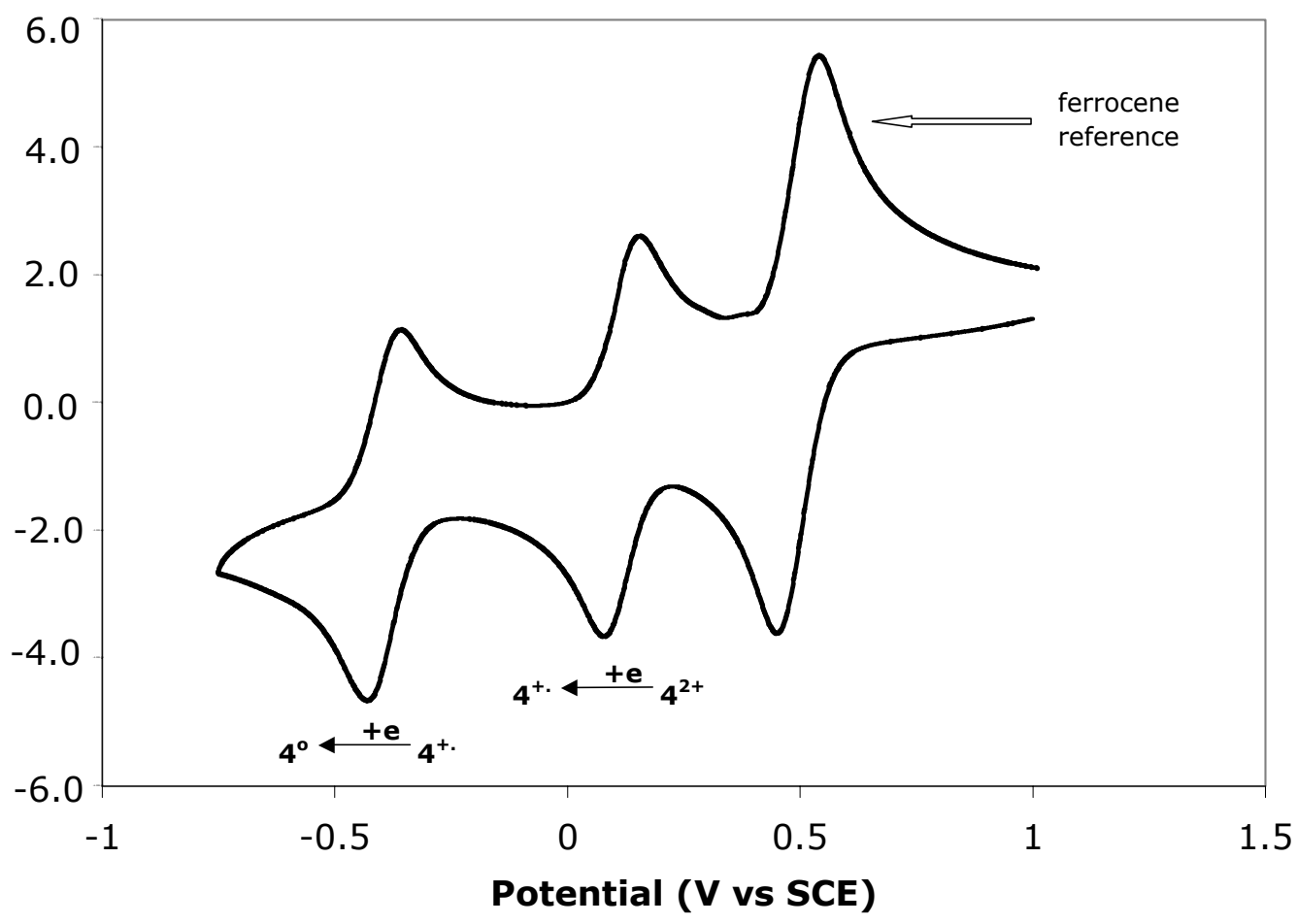

Figure 2. Cyclic Voltammogram for $4^{2+}$ in acetonitrile

A substantial anodic current for oxidation of the pyrylogen radical cations in oxygen saturated acetonitrile solutions was observed even at scan rates of $50 \mathrm{mV} / \mathrm{sec}$ reflecting their reluctance to react with oxygen. This is in dramatic contrast to the rapid reaction of $2 \bullet(\mathrm{Ar}=\mathrm{Ph})$, which does not survive for oxidation to the dication in the presence of oxygen, even at scan rates of $500 \mathrm{mV} / \mathrm{sec}$. We suggest that the reduced reactivity of the pyrylogen radical cations is a consequence of their positive charge coupled with the electrophilic character of oxygen and the increased spin delocalization in the pyrylogen radical cations in comparison to $2 \bullet(\mathrm{Ar}=\mathrm{Ph})$.

Computational evidence for energy minima corresponding to all three partners in this redox system as depicted in Figure 2 was obtained using the B3LYP/6-31G(d) model. Table 1 lists the computed bond lengths connecting the two rings, $\mathrm{d}_{44}$, the ring-ring dihedral angle, $>344^{\prime} 3^{\prime}$, and these values measured in the crystal structures of $\mathbf{1}^{2+}(\mathrm{Ar}=\mathrm{Ph}) \cdot 2 \mathrm{~B}\left(\mathrm{C}_{6} \mathrm{~F}_{5}\right)_{4}$ and $\mathbf{4}^{\mathbf{2 +}}$. Frequency calculations were performed to verify location of energy minima. The absence of spin contamination was verified in each calculation by examination of $\left\langle S^{2}\right\rangle$, which showed values acceptably close to 0 for all singlets and to 0.75 for all doublets. The inter-ring distance, $\mathrm{d}_{44}$, and the dihedral angle between the pyridinium and pyrylium rings both decrease along the series dication $>$ radical cation $>$ neutral consistent with an increasing 4-4' bond order. The inter-ring "double-bond" in the neutral redox partner is nearly planar as implied by the structure drawn in Figure 2. The absence of a planar aromatic pyridinium ring is also evident from the distinct bending of the $\mathrm{N}$-methyl group out of the plane of the ring. This distortion $\left(>\mathbf{3}^{\prime} \mathbf{2}^{\prime} \mathbf{N C H}_{3}>\right.$, 
increases along the series dication $<$ radical cation $<$ neutral. The computed and X-Ray bond lengths in the dications are in reasonable agreement although slightly shorter $\mathrm{d}_{44}$, distances were observed experimentally. The computed and X-Ray dihedral angles, however, were significantly different perhaps reflecting counterion interactions (vide supra) or crystal packing forces.

Table 1. B3LYP/6-31G(d) Structural data for the 1 and 4 Redox Partners and X-Ray Data for the dications.

\begin{tabular}{|c|c|c|c|c|}
\hline & Energy (hartrees) & $\mathrm{d}_{44}$, & $>344^{\prime} 3^{\prime}$ & $>3^{\prime} 2^{\prime} \mathrm{NCH} 3$ \\
\hline $1^{2+} \cdot 2 \mathrm{~B}\left(\mathrm{C}_{6} \mathrm{~F}_{5}\right)_{4}{ }^{\mathrm{b}}$ & & $1.474 \AA$ & $53.23^{\circ}$ & $179.57^{\circ}$ \\
\hline $1^{2+}$ & -1016.971268 & $1.487 \AA$ & $41.50^{\circ}$ & $178.19^{\circ}$ \\
\hline $1^{+} \bullet$ & -1017.287146 & $1.435 \AA$ & $3.84^{\mathrm{o}}$ & $176.94^{\circ}$ \\
\hline $\mathbf{1}^{0}$ & -1017.465607 & $1.390 \AA$ & $0.75^{\circ}$ & $169.56^{\circ}$ \\
\hline $4^{2+} \cdot 2 \mathrm{BF}_{4}{ }^{-b}$ & & $1.482 \AA$ & $29.99^{\circ}$ & $176.42^{\circ}$ \\
\hline $4^{2+}$ & -869.296220 & $1.490 \AA$ & $41.40^{\circ}$ & $178.24^{\mathrm{o}}$ \\
\hline $4^{+} \bullet$ & -869.624817 & $1.433 \AA$ & $1.64^{\circ}$ & $176.92^{\circ}$ \\
\hline $4^{0}$ & -869.800536 & $1.384 \AA$ & $0.17^{\circ}$ & $166.94^{\circ}$ \\
\hline
\end{tabular}

a. ZPE corrected. ${ }^{\text {b. }}$ X-ray crystallography data.

Reduction of $\mathbf{1}^{\mathbf{2}}(\mathrm{Ar}=\mathrm{Ph}) \cdot 2 \mathrm{BF}_{4}^{-}$and $\mathbf{4}^{\mathbf{2 +}}$ with zinc dust in an inert atmosphere resulted in highly colored solutions. The UV-Vis spectra of these solutions are very similar with prominent peaks at $382 \mathrm{~nm}$ and $385 \mathrm{~nm}$ and broad bands with resolvable fine structure at $563 \mathrm{~nm}$ and 614 $\mathrm{nm}$ for the $\mathbf{4}^{\mathbf{2 +}}$ and $\mathbf{1}^{\mathbf{2}}(\mathrm{Ar}=\mathrm{Ph}) \cdot 2 \mathrm{BF}_{4}{ }^{-}$reaction mixtures, respectively. (See Figure 3 for the UVVis spectrum for the $\mathbf{4}^{2+}$ reaction mixture) The same UV-Vis spectrum was produced by Zn dust reduction and coulometric reduction of $\mathbf{1}^{2+}(\mathrm{Ar}=\mathrm{Ph}) \cdot 2 \mathrm{BF}_{4}{ }^{-}$at a potential capable of making the radical cation but not the neutral redox partner. In addition, the $\mathbf{1}^{2+}(\mathrm{Ar}=\mathrm{Ph}) \cdot 2 \mathrm{BF}_{4}{ }^{-}$reaction mixture produced a broad single line ESR signal with a g-value of 2.0215 . These results suggest that the color materials are the radical cations. This suggestion is also supported by time-domain density functional theory calculations in $\mathrm{CH}_{3} \mathrm{CN}^{14}$ that predict that the radical cation of $\mathbf{4}^{2+}$ should absorb at $506 \mathrm{~nm}$ while the neutral redox partner, $\mathbf{4}^{\mathbf{0}}$, should absorb at $355 \mathrm{~nm}$. The radical cations appear to be indefinitely stable in an inert atmosphere. However, despite their stability on the cyclic voltammetry timescale, they do react on the longer laboratory timescale with oxygen. 


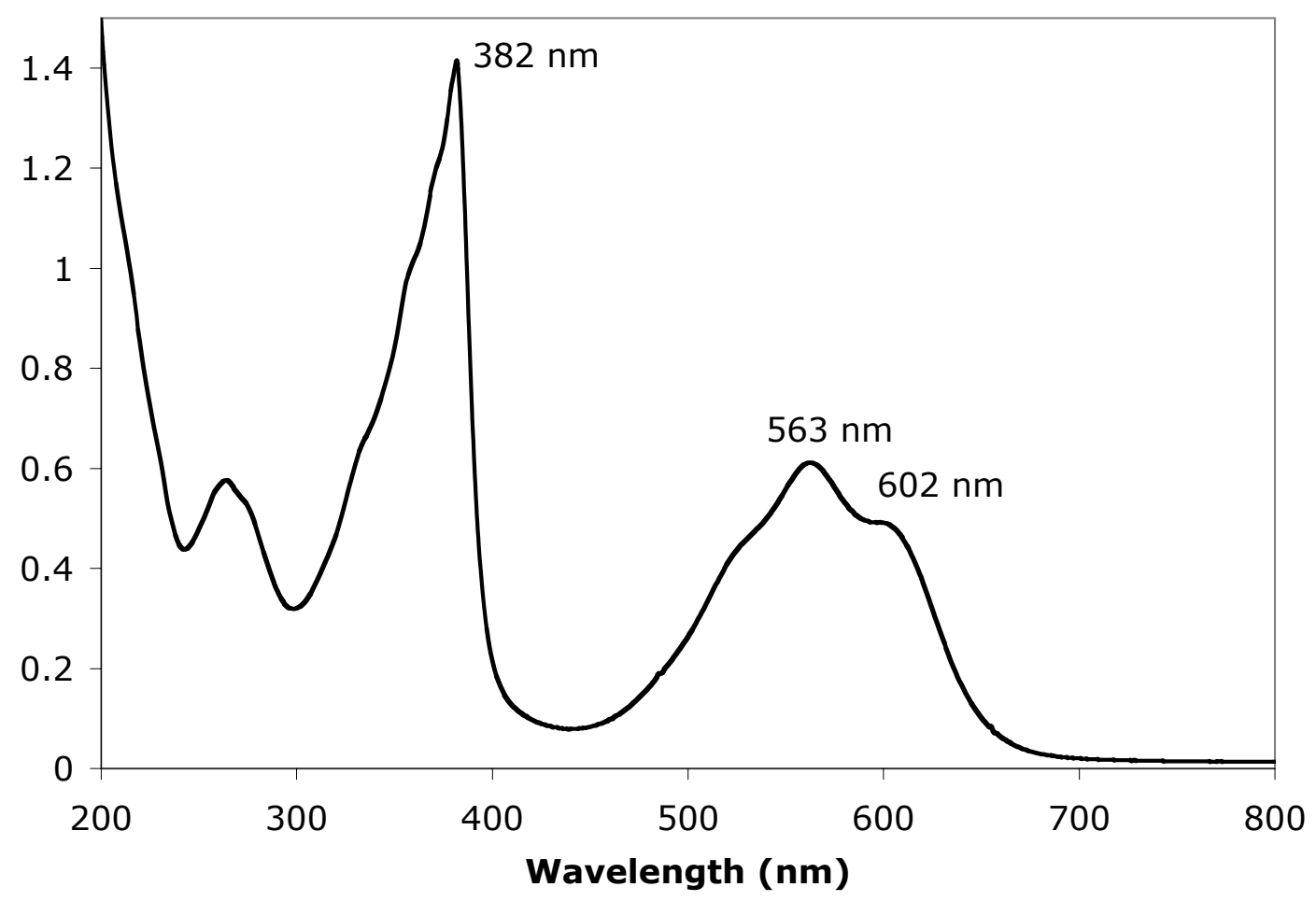

Figure 3. UV-Visible spectrum of the product from reduction of $\mathbf{4}^{\mathbf{2 +}}$ with $\mathrm{Zn}$ dust.

\section{Photophysical data}

The photophysical data for $\mathbf{1}^{\mathbf{2 +}}(\mathrm{Ar}=\mathrm{Ph}) \cdot 2 \mathrm{BF}_{4}^{-}, \mathbf{4}^{\mathbf{2 +}}$, and $\mathbf{2}^{+}$are compared in Table 2 . The UVVisible spectra of $\mathbf{1}^{\mathbf{2}}(\mathrm{Ar}=\mathrm{Ph}) \cdot 2 \mathrm{BF}_{4}{ }^{-}$and $\mathbf{2}^{+}$are very similar and exhibit three peaks at energies lower than $250 \mathrm{~nm}$. The UV-Visible spectrum of $\mathbf{2}^{+}$has been described in terms of two chromophores one in which the transition dipole moment is polarized in the X-direction and the other in the Y-direction. ${ }^{15}$ (See Figure 4) This concept of two perpendicular chromophores for $\mathbf{2}^{+}$ is supported by time domain density functional calculations. The two lowest energy transitions are calculated to appear at $408 \mathrm{~nm}$ and $375 \mathrm{~nm}$ in $\mathrm{CH}_{3} \mathrm{CN}$ using the polarization continuum model. The lowest energy transition corresponds to promotion of an electron from the HOMO that is localized along the $\mathrm{X}$-axis to the LUMO and corresponds to the X-band. The next lowest energy transition occurs from HOMO-1, which is heavily localized in the 4-phenyl ring (i.e. along the y-axis) to the LUMO and consequently corresponds to the charge-transfer Y-band. 


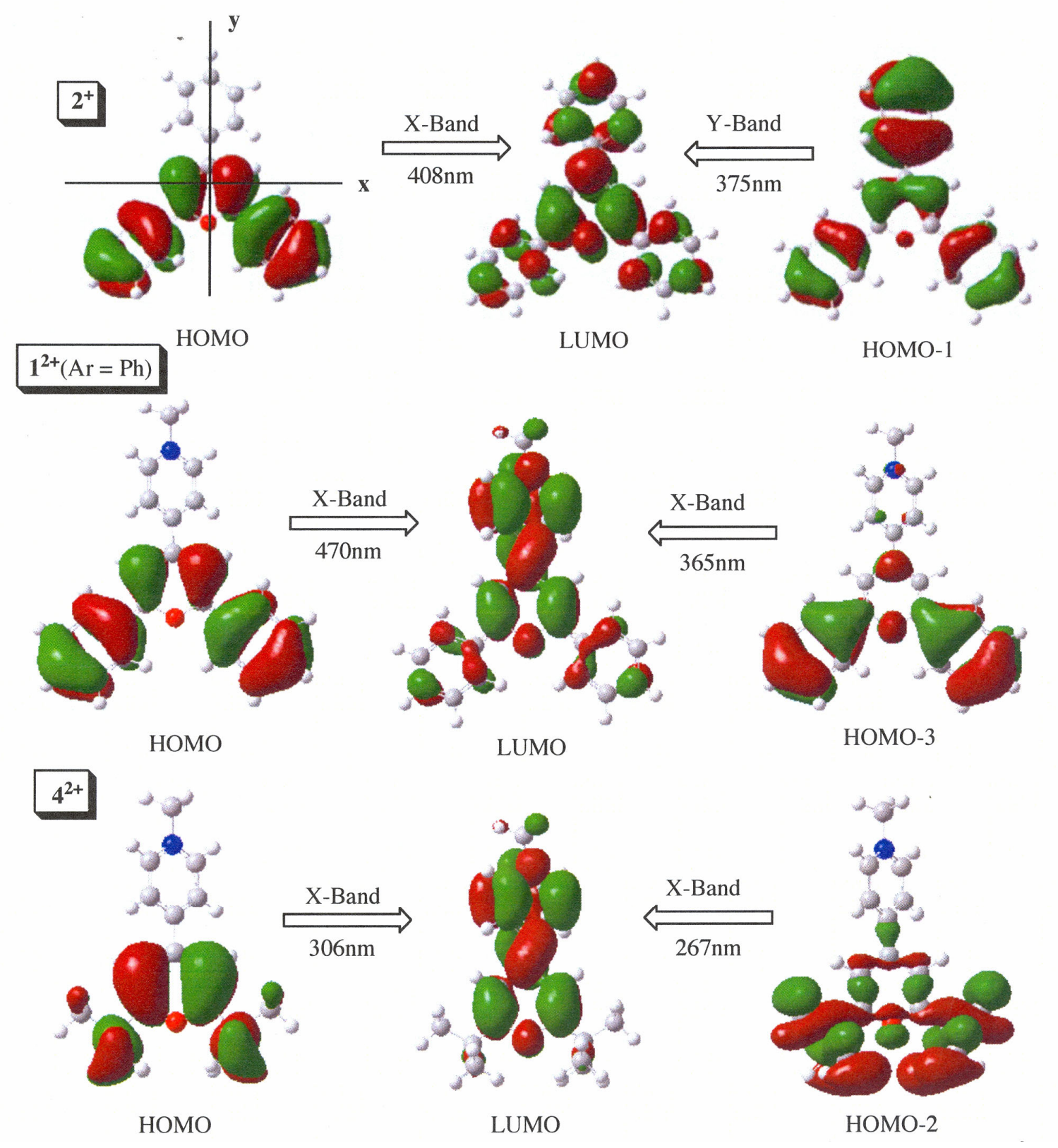

Figure 4. Calculated low energy transitions in the UV-Vis spectra of $\mathbf{2}^{+}, \mathbf{1}^{2+}(\mathrm{Ar}=\mathrm{Ph})$, and $\mathbf{4}^{2+}$.

The charge-transfer Y-band is missing in the UV-visible spectra of $\mathbf{1}^{2+}(\mathrm{Ar}=\mathrm{Ph}) \bullet 2 \mathrm{BF}_{4}^{-}$, and $4^{2+}$. (See Figure 4) This is a consequence of the electron poor character of the pyridinium ring that makes electron transfer to the pyrylium ring energetically inaccessible. The lowest energy transition in the pyrylogens involves promotion of an electron from the HOMO to the LUMO comparable to the X-band in $\mathbf{2}^{+}$. The next lowest transition corresponds is the HOMO$3 \rightarrow$ LUMO and HOMO-2 $\rightarrow$ LUMO excitation in $\mathbf{1}^{2+}(\mathrm{Ar}=\mathrm{Ph}) \bullet 2 \mathrm{BF}_{4}{ }^{-}$and $\mathbf{4}^{2+}$, respectively, that is best characterized as another X-band transition. (Figure 4) The replacement of the phenyl 
groups in $\mathbf{1}^{\mathbf{2}}(\mathrm{Ar}=\mathrm{Ph}) \cdot 2 \mathrm{BF}_{4}{ }^{-}$by the tert-butyl groups in $\mathbf{4}^{2+}$ decreases the extent of the $\pi$-system and generates a significant hypsochromic shift $(128 \mathrm{~nm})$ and reduction in the extinction coefficient $(31,000 \rightarrow 8,000)$.

Table 2. Photophysical data for pyrylogens

\begin{tabular}{llll}
\hline & $\mathbf{1}^{\mathbf{2 +}}(\mathrm{Ar}=\mathrm{Ph}) \cdot 2 \mathrm{BF}_{4}{ }^{-}$ & $\mathbf{4}^{\mathbf{2 +}}$ & $\mathbf{2}^{+}$ \\
\hline$\lambda_{\mathrm{MAX}}(\varepsilon)^{\mathrm{a}}$ & $281(45,000)$ & $267(18,000)$ & $277(18,000)$ \\
& $327(16,000)$ & $312(8,000)$ & $355(32,000)$ \\
$\lambda_{\mathrm{F}}{ }^{\mathrm{b}}$ & $440(31,000)$ & & $401(25,000)$ \\
$\lambda_{\mathrm{P}}{ }^{\mathrm{c}}$ & 533 & 402 & 465 \\
$\tau_{\mathrm{P}}{ }^{\mathrm{d}}$ & 565 & 456 & 520 \\
$\mathrm{E}\left(\mathrm{S}_{1}\right)^{\mathrm{f}}$ & $35.6 \pm 0.2$ & $29.8 \pm 0.8^{\mathrm{e}}$ & $225 \pm 3$ \\
$\mathrm{E}\left(\mathrm{T}_{1}\right)^{\mathrm{f}}$ & 59 & 80 & 65 \\
$\Phi_{\mathrm{F}}{ }^{\mathrm{g}}$ & 54 & 70 & 53 \\
\hline
\end{tabular}

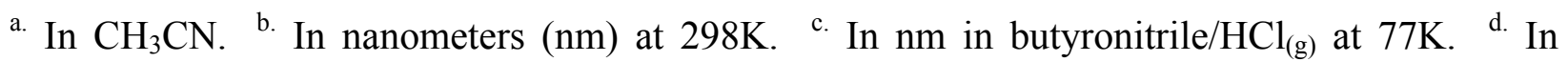
milliseconds $(\mathrm{ms})$ at $77 \mathrm{~K}$ in ethanol/ $\mathrm{HCl}_{(\mathrm{g})}$. ${ }^{\text {e. }}$ In milliseconds $(\mathrm{ms})$ at $77 \mathrm{~K}$ in butyronitrile $/ \mathrm{HCl}_{(\mathrm{g})}$. f. In kcal/mol. g. Fluorescence quantum yield in $\mathrm{CH}_{3} \mathrm{CN}$.

Pyrylogens $\mathbf{1}^{2+}(\mathrm{Ar}=\mathrm{Ph}) \cdot 2 \mathrm{BF}_{4}{ }^{-}$and $\mathbf{4}^{\mathbf{2 +}}$ both fluoresce and phosphoresce at the wavelengths given in Table 2. The quantum yield of fluorescence for $\mathbf{1}^{2+}(\mathrm{Ar}=\mathrm{Ph}) \cdot 2 \mathrm{BF}_{4}{ }^{-}$was nearly 8 times greater than for $4^{2+}$. In addition, although we didn't measure the quantum yield, the intensity of phosphorescence was also significantly larger for $\mathbf{1}^{2+}(\mathrm{Ar}=\mathrm{Ph}) \cdot 2 \mathrm{BF}_{4}{ }^{-}$than for $\mathbf{4}^{\mathbf{2 +}}$ suggesting that radiationless deactivations of the excited states of the latter compound are more important. The triplet-triplet absorption spectrum of $1^{2+}(\mathrm{Ar}=\mathrm{Ph}) \cdot 2 \mathrm{BF}_{4}^{-}\left(\lambda_{\mathrm{MAX}}=570 \mathrm{~nm}\right)$ was observed in a nanosecond laser flash experiment upon irradiation at $355 \mathrm{~nm}$ with the $3^{\text {rd }}$ harmonic of a $\mathrm{Nd}$ :YAG laser. The assignment to the triplet was made by comparison to the absorption spectrum of the corresponding radical cation and with the observation that the transient absorption spectrum was effectively quenched by oxygen. Attempts to observed the triplet-triplet absorption spectrum of $\mathbf{4}^{\mathbf{2 +}}$ failed. The radical cation of $\mathbf{4 2 +}$ rather than the triplet was observed even at laser powers as low as $3 \mathrm{~mJ} /$ pulse. The very high singlet and triplet energies of $\mathbf{4}^{2+}$ (Table 2) coupled with its ease of reduction $(+0.07 \mathrm{~V}$ vs SCE) leads to reduction potentials of $3.54 \mathrm{eV}$ and $3.13 \mathrm{eV}$ for the singlet and triplet excited states, respectively. Since the work necessary ($\mathrm{e}_{\mathrm{o}}{ }^{2} /(\varepsilon a)$ to bring two radical cations to the encounter distance $a$ in a solvent of dielectric constant, $\varepsilon$, is only $-55 \mathrm{mV}$ in $\mathrm{CH}_{3} \mathrm{CN}$ at a typical separation distance of $7 \AA$ these excited states are potent oxidants capable of oxidizing all substrates with oxidation potentials less than $3.48 \mathrm{~V}$ (singlet) and $3.07 \mathrm{~V}$ (triplet). The fluorescence of $\mathbf{4}^{\mathbf{2 +}}$ was quenched by p-xylene with a Stern Volmer $\mathrm{K}_{\mathrm{SV}}$ =34. (Figure 5) The low intensity of the fluorescence precluded measurement of the singlet lifetime for $\mathbf{4}^{2+}$ but a value of $3.42 \pm 0.23$ ns was obtained for $\mathbf{1}^{2+}(\mathrm{Ar}=\mathrm{Ph}) \cdot 2 \mathrm{BF}_{4}{ }^{-9}$. 


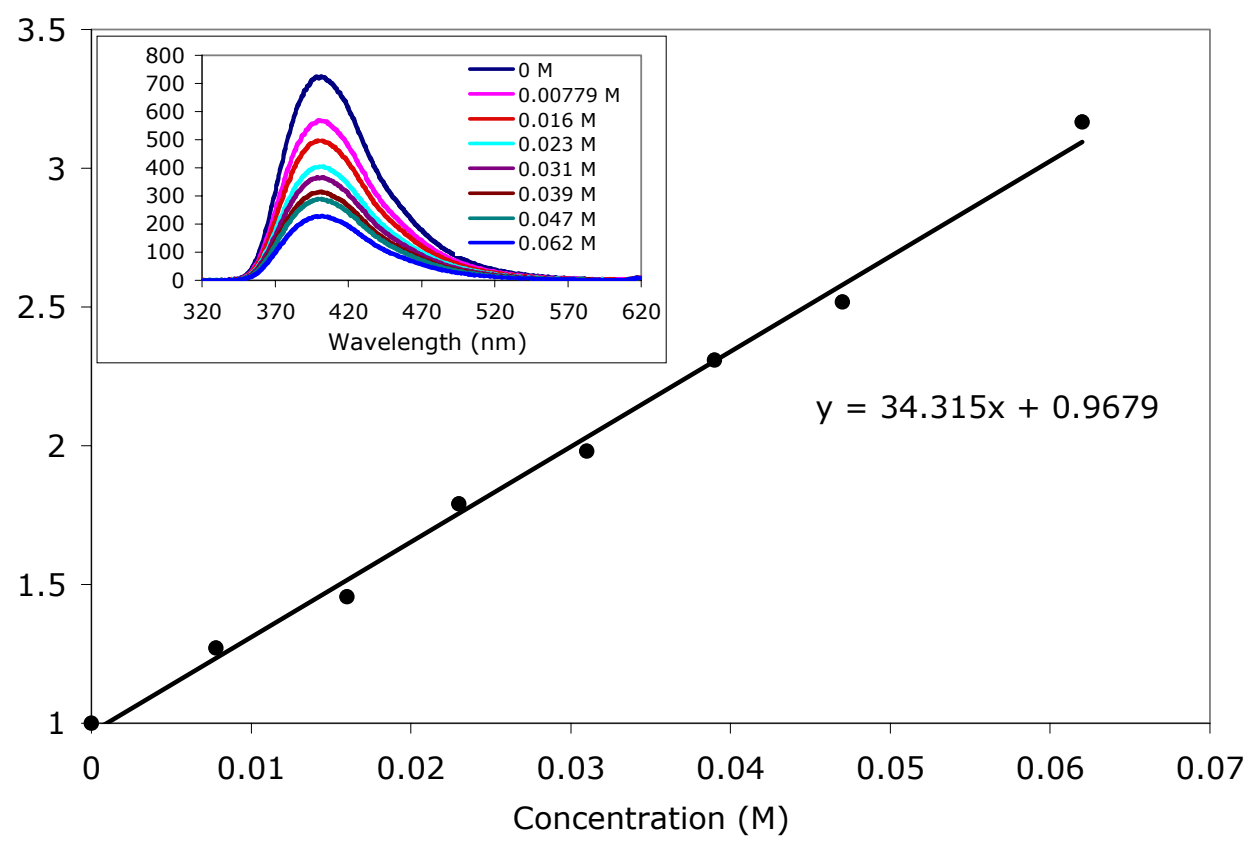

Figure 5. Stern Volmer Plot for Quenching of $\mathbf{4}^{2+}$ with p-Xylene.

\section{Solubility data}

The maximum solubility of $\mathbf{4}^{\mathbf{2}}$ increases with the dielectric constant $(\varepsilon)$ of the solvent (solvent, $\varepsilon$, maximum solubility-M; chloroform, 4.81, $7.59 \times 10^{-6} \mathrm{M}$; tetrahydrofuran, 7.52, $2.14 \times 10^{-5} \mathrm{M}$; dichloromethane, 8.93, $2.15 \times 10^{-4} \mathrm{M}$; 1,2-dichloroethane, $10.42,3.02 \times 10^{-4} \mathrm{M}$; acetonitrile, $36.64,0.05 \mathrm{M}$ ). The solubility is sufficient (Absorbance $>0.3$ at $312 \mathrm{~nm}$ ) in acetonitrile, 1,2dichloroethane, and dichloromethane, but insufficient in tetrahydrofuran and chloroform for use in photochemical electron transfer reactions. Pyrylogen $\mathbf{1}^{\mathbf{2 +}}(\mathrm{Ar}=\mathrm{Ph}) \cdot 2 \mathrm{BF}_{4}{ }^{-}$is significantly less soluble than $\mathbf{4}^{2+}$ and only usable as a sensitizer in acetonitrile $(0.014 \mathrm{M})$ and 1,2-dichloroethane $\left(1.8 \times 10^{-5} \mathrm{M}\right)$. It has a very low solubility in dichloromethane $\left(6.2 \times 10^{-6} \mathrm{M}\right)$ and no solubility in THF or chloroform.

\section{Conclusions}

The dialkyl substituted pyrylogen, $4^{2+}$ shows dramatically enhanced solubility in comparison to its diphenyl analogue, $\mathbf{1}^{\mathbf{2 +}}(\mathrm{Ar}=\mathrm{Ph}) \cdot 2 \mathrm{BF}_{4}{ }^{-}$in acetonitrile, ethereal, and halocarbon solvents. It also forms stable radical cation and neutral redox partners. The reduction potentials coupled with the exceptionally high excited state energies allow characterization of this new sensitizer as a very potent oxidant. Unfortunately, it's significant hypsochromatic shift relative to $\mathbf{1}^{\mathbf{2}}(\mathrm{Ar}=$ $\mathrm{Ph}) \bullet 2 \mathrm{BF}_{4}^{-}$is likely to decrease its general utility as a new photosensitizer. 


\section{Experimental Section}

General Procedures. IR spectra were recorded on a Nicolet Nexus 470 FT-IR. The ESR was recorded on a Bruker EMX (X-band) ESR. Laser flash photolysis data was collected using a Spectra Physics 10Hz INDI-40 Nd:YAG laser coupled to a Luzchem nanosecond laser flash photolysis optical bench. ElectroSpray Ionization mass spectra and MS-MS spectra were obtained with a Thermo-Finnigan LCQ mass spectrometer. Proton and carbon NMR were obtained on a Bruker $400 \mathrm{MHz}$ NMR and are referenced to TMS. Phosphorescence and fluorescence spectra were recorded on a Cary Eclipse Fluoresce spectrophotometer. Cyclic Voltammograms were collected with a $\mathrm{CH}$ Instruments $\mathrm{CHI600C}$ Electrochemical Analyzer. UV-Visible spectra were collected with the Agilent 8453 UV-visible spectrophotometer. Sodium hydride (95\%), pinacolone (97\%), pyridine-4-carboxaldehyde (97\%), trimethyloxonium tetrafluoroborate (95\%), triphenyl methanol (97\%), tetrafluoroboric acid $(48 \%$ by weight in water), and 1,2-dichloroethane (sure seal spectroscopic grade) were all obtained from Aldrich and used without further purification. Baker analyzed acetic anhydride (97.2\%, and Fluka butyronitrile (99\%) were distilled prior to use. Fisher HPLC grade acetonitrile (99.9\%) was distilled and stored over activated 3A molecular sieves.

Cyclic voltammetry. An acetonitrile solution $2 \times 10^{-3} \mathrm{M}$ in substrate, $0.1 \mathrm{M}$ in $\mathrm{Bu}_{4} \mathrm{~N}^{+} \mathrm{ClO}_{4}^{-}$, and $2 \times 10^{-2} \mathrm{M}$ ferrocene as an internal standard was placed in a flask containing a glassy carbon electrode, a non-aqueous reference electrode $\left(\mathrm{Ag} / \mathrm{AgNO}_{3}\left(0.01 \mathrm{M}\right.\right.$ in $\left.\mathrm{CH}_{3} \mathrm{CN}\right)$, and a platinium wire electrode. The solution was deoxygenated by bubbling with argon prior to the electrochemical experiment.

Fluorescence quantum yields. The method of Eaton ${ }^{16}$ was used to measure fluorescence quantum yields. The fluorescence intensity of the pyrylogens were collected in acetonitrile and compared to that of 9, 10-diphenylanthracene in cyclohexane $\left(\Phi_{\mathrm{f}}=0.7 \pm 0.04\right) .{ }^{17}$ The quantum yields, $\Phi_{\mathrm{PY}}$ were measured three times and calculated using the following equation which contains the values for the refractive index of cyclohexane $\left(\mathrm{n}_{\mathrm{s}}=1.4262\right)$ and acetonitrile $(\mathrm{n}=$ 1.3441), the integrated intensities across the emission bands of 9,10-diphenylanthracene $\left(\mathrm{F}_{\mathrm{s}}\right)$ and the pyrylogen $\left(\mathrm{F}_{\mathrm{Py}}\right)$, the absorbances at the excitation wavelengths for 9.10-diphenylanthracene $\left(A_{s}\right)$ and the pyrylogen $\left(A_{P y}\right)$, and the fluorescence quantum yield of 9, 10-diphenylanthracene, $\Phi_{\mathrm{S}}$.

$$
\Phi_{P Y}=\frac{A_{S} F_{P Y} n^{2}}{A_{P Y} F_{S} n_{S}{ }^{2}} \Phi_{S}
$$

Computational Studies. Calculations were performed with the Gaussian 03 program $^{18}$ using the Becke/Stephens ${ }^{19,20}$ three parameter Lee-Yang-Parr correlation hybrid functional B3LYP in 
conjunction with the 6-31G(d) basis set. Frequency calculations were used to verify location of energy minima. The absence of spin contamination was verified in each calculation by examination of $\left\langle\mathrm{S}^{2}>\right.$ which showed values acceptably close to 0 for all singlets and to 0.75 for all doublets. $^{21}$

Zn dust reductions. A $3 \mathrm{~mL}$ acetonitrile solution $7.81 \times 10^{-5} \mathrm{M}$ in $4^{2+}$ was placed into a side arm attached to a quartz cuvette. This solution was then subjected to three freeze-pump-thaw cycles and then tilted in order to pour the solution into the cuvette arm containing $38 \mathrm{mg}\left(5.81 \times 10^{-4} \mathrm{M}\right)$ of zinc dust. The solution turned purple within one minute and the color continued to intensify for 4 hours and then ceased signaling completion of the reaction. The UV-visible spectrum depicted in Figure 3 was then taken. The color was maintained indefinitely but rapidly disappeared when the cuvette was opened to the atmosphere.

Crystallographic data. The X-ray diffraction data were measured at $150 \mathrm{~K}$ on a Bruker SMART APEX II CCD area detector system equipped with a graphite monochromator and a Mo $\mathrm{K} \alpha$ fine-focus sealed tube operated at $1.50 \mathrm{~kW}$ power $(50 \mathrm{kV}, 30 \mathrm{~mA})$. A colorless rectangular prismatic crystal of approximate dimensions $0.42 \mathrm{~mm} \times 0.21 \mathrm{~mm} \times 0.18 \mathrm{~mm}$ glued to a MiTeGen micromount using Paratone $\mathrm{N}$ oil. The detector was placed at a distance of $5.9 \mathrm{~cm}$ from the crystal during the data collection.

A series of narrow frames of data were collected with a scan width of $0.5^{\circ}$ in $\omega$ or $\phi$ and an exposure time of $10 \mathrm{~s}$ per frame. The frames were integrated with the Bruker SAINT Software package $2^{22}$ using a narrow-frame integration algorithm. The integration of the data using a monoclinic unit cell yielded a total of 38265 reflections to a maximum $2 \theta$ angle of $66.28^{\circ}$ of which 8315 were independent with $I \geq 2 \sigma(I)\left(\mathrm{R}_{\text {int }}=0.0307\right)$. The data were corrected for absorption effects by the multi-scan method (SADABS). Crystallographic data collection parameters and refinement data are collected below in Table 1. The structure was solved by direct methods using the Bruker Software Package. All atoms were located in successive Fourier maps. Whereas the non-hydrogens were refined anisotropically, the hydrogen atoms were refined isotropically. The final refinement parameters are $R_{l}=0.0409$ and $w R 2=0.1084$ for data with $F$ $>4 \sigma(F)$ giving the data to parameter ratio of 21. The refinement data for all data are $R_{1}=0.0539$ and $w R 2=0.1169$.

[t-BuPy] $\left(\mathrm{BF}_{4}\right)_{2}$ crystallizes in the monoclinic space group $P 2_{1} / n$. The asymmetric unit consists of a t-BuPy dication and two tetrafluoroborate anions. Both ions are well ordered. The dication is non-planar as the pyrilium and pyridinium rings are twisted at $29.99(4)^{\circ}$. Both anions are located near the pyrylium $\mathrm{O}$ atom of the dication with the associated $\mathrm{O}(1) \cdots \mathrm{F}(1)$ and $\mathrm{O}(1) \cdots \mathrm{F}(7)$ distances of 2.8481(9) and 3.0697(9) $\AA$, respectively, revealing moderately strong inter-ionic interactions.

\section{Compound characterization}


1,5-Di-tert-butyl-3-(4-pyridyl)pentane-1,5-dione (5). Sodium hydride (75 mg, $3.125 \mathrm{mmol}$ ) was added under a nitrogen atmosphere to a $20 \mathrm{~mL}$ flask submerged in a ice bath containing $3 \mathrm{~mL}$ of DMSO. After the addition was complete an additional aliquot of DMSO (1 mL) was added and the mixture allowed to stir for $15 \mathrm{~min}$. Pinacolone $(1.9 \mathrm{~g}, 18.97 \mathrm{mmol})$ was then added and the reaction vessel was removed from the ice bath. This solution was then allowed to stir for $30 \mathrm{~min}$ followed by dropwise addition over a $10 \mathrm{~min}$ period of pyridine-4-carboxaldehyde $(1.0 \mathrm{~g}$, $9.34 \mathrm{mmol}$ ). An additional $1 \mathrm{~mL}$ aliquot of DMSO was added and the reaction mixture stirred for $30 \mathrm{~min}$. The reaction mixture was then poured into $20 \mathrm{~mL}$ of water and the resulting solid was filter, washed with copious amounts of water, and air dried. This material was then recrystallized from ethanol/water and dried to get $1.27 \mathrm{~g}(4.388 \mathrm{mmol}, 47 \%)$ of product. IR (KBr pellet; $\mathrm{cm}^{-1}$ ) 2973, 1705. ${ }^{1} \mathrm{H}$ NMR $\left(\mathrm{CDCl}_{3}\right) \delta 1.05(\mathrm{~s}, 18 \mathrm{H}), 2.82(\mathrm{dd}, \mathrm{J}=6.5,17.7 \mathrm{~Hz}, 2 \mathrm{H}), 2.87(\mathrm{dd}, \mathrm{J}=7.5$, $17.7 \mathrm{~Hz}, 2 \mathrm{H}), 3.73$ (pentuplet, $\mathrm{J}=6.9 \mathrm{~Hz}, 1 \mathrm{H}), 7.14-7.16(\mathrm{~m}, 2 \mathrm{H}), 8.47-8.49(\mathrm{~m}, 2 \mathrm{H}) .{ }^{13} \mathrm{C}$ NMR $\left(\mathrm{CDCl}_{3}\right) \delta 26.28,35.41,41.76,44.17,123.10,149.95,153.66,213.35$.

$\mathrm{N}$-Methyl-1,5-di-tert-butyl-3-(4-pyridyl)pentane-1,5-dione tetrafluoroborate (6). A solution of 5 (578 mg, $2.1 \mathrm{mmol})$ and trimethyloxonium tetrafluoroborate $(312 \mathrm{mg}, 2.1 \mathrm{mmol})$ in $2.5 \mathrm{~mL}$ of $\mathrm{CHCl}_{3}$ was stirred at room temperature for $30 \mathrm{~min}$. The solvent was then removed by rotoevaporation, the residue suspended in $25 \mathrm{~mL}$ of ethyl ether and filtered. Recrystallization from ethanol afforded a white crystalline product (469 mg, 60\%). IR (KBr pellet; $\mathrm{cm}^{-1}$ ) 2970, 1702. ${ }^{1} \mathrm{H}$ NMR $\left(\mathrm{CDCl}_{3}\right) \delta 1.07(\mathrm{~s}, 18 \mathrm{H}), 3.03(\mathrm{dd}, \mathrm{J}=5.6,18.7 \mathrm{~Hz}, 2 \mathrm{H}), 3.12(\mathrm{dd}, \mathrm{J}=8.3,18.7$ $\mathrm{Hz}, 2 \mathrm{H}), 3.79-3.87(\mathrm{~m}, 1 \mathrm{H}), 4.54(\mathrm{~s}, 3 \mathrm{H}), 7.98(\mathrm{~d}, \mathrm{~J}=6.5 \mathrm{~Hz}, 2 \mathrm{H}), 8.59(\mathrm{~d}, \mathrm{~J}=6.5 \mathrm{~Hz}, 2 \mathrm{H}) .{ }^{13} \mathrm{C}$ NMR $\left(\mathrm{CDCl}_{3}\right) \delta 26.18,36.27,41.46,43.94,47.79,127.76,144.19,166.24,213.67$.

$\mathbf{N}$-Methyl-2,6-di-tert-butyl-4,4'-pyrylogen bis-tetrafluoroborate $\left(\mathbf{4}^{\mathbf{2 +}}\right)$. Acetic anhydride $(3.5 \mathrm{~mL})$ was added to a mixture of $6(136.5 \mathrm{mg}, 0.35 \mathrm{mmol})$ and triphenyl methanol $(197.5 \mathrm{mg}$, $0.758 \mathrm{mmol})$ under a nitrogen atmosphere. The solution was gently heated with a warm water bath $\left(\sim 50^{\circ} \mathrm{C}\right)$ to dissolve the solids and then cooled to room temperature. Tetrafluoroboric acid ( $278 \mu \mathrm{L}, 48 \%$ in $\mathrm{H}_{2} \mathrm{O}$, equivalent to $133 \mathrm{mg}, 1.51 \mathrm{mmol}$ of $\mathrm{HBF}_{4}$ ) was added and the mixture heated to $50^{\circ} \mathrm{C}$ for $30 \mathrm{~min}$. The product was induced to crystallize by the addition of diethylether. The crude product was recrystallized from acetic acid washed with diethylether and dichloromethane to give $114 \mathrm{mg}(71.4 \%)$ of colorless crystals after drying. IR (KBr pellet; $\mathrm{cm}^{-}$ 1) $3136,2980,1633,1531,1076 .{ }^{1} \mathrm{H} \mathrm{NMR}\left(\mathrm{CD}_{3} \mathrm{CN}\right) \delta 1.67(\mathrm{~s}, 18 \mathrm{H}), 4.40(\mathrm{~s}, 3 \mathrm{H}), 8.30(\mathrm{~s}, 2 \mathrm{H})$, $8.49(\mathrm{~d}, \mathrm{~J}=5.9 \mathrm{~Hz}, 2 \mathrm{H}), 8.93(\mathrm{~d}, \mathrm{~J}=5.93 \mathrm{H}) .{ }^{13} \mathrm{C} \mathrm{NMR}\left(\mathrm{CD}_{3} \mathrm{CN}\right) \delta 28.45,40.84,49.90,119.81$, $128.59,147.76,149.58,162.42,190.69$.

\section{Supplementary Information Available}

Computational data for $\mathbf{4}^{2+}$ and its redox partners, ES/MS spectra for $\mathbf{4}^{2+}$ and CIF file for X-Ray structure of $4^{2+}$.

\section{Acknowledgements}


We thank the National Science Foundation for their generous support of this research and Dr. Navamoney Arulsamy for collection of the X-Ray diffraction data.

\section{References}

1. SciFinder Scholar. In American Chemical Society: 2006.

2. There are examples of singlet and triplet ion pairs with the same or similar RET efficiencies see: Roth, H. D., J. Phys. Chem. A 2003, 107, 3432.

3. Mattes, S. L.; Farid, S. J. Am. Chem. Soc. 1986, 108, 7356.

4. Kavarnos, G. J. In Fundamentals of Photoinduced Electron Transfer. VCH Publishers Inc.: New York, N.Y., 1993; p 359.

5. Gould, I. R.; Ege, D.; Moser, J. E.; Farid, S. J. Am. Chem. Soc. 1990, 112, 4290.

6. Gould, I. R.; Moser, J. E.; Armitage, B.; Farid, S. Res. Chem. Intermed. 1995, 21, 793.

7. Gould, I. R.; Farid, S. J. Phys. Chem. 1993, 97, 13067.

8. Miranda, M. A. Chem. Rev. 1994, 94, 1063.

9. Clennan, E. L.; Liao, C.; Ayokosok, E. J. Am. Chem. Soc. 2008, 130, 7552.

10. Suga, K.; Ohkubo, K.; Fukuzumi, S. J. Phys. Chem. A 2005, 109, 10168.

11. Fukuzumi, S.; Ohkubo, K.; Suenobu, T.; Kato, K.; Fujitsuka, M.; Ito, O. J. Am. Chem. Soc. 2001, 123, 8459.

12. Marcus, R. A. J. Chem. Phys. 1956, 24, 966.

13. Marcus, R. A., Chemical and electrochemical electron-transfer theory. In Ann. Rev. Phys. Chem., Eyring, H., Ed. 1964; Vol. 15, pp 155-196.

14. Petersilka, M.; Grossmann, U. J.; Gross, E. K. U. Phys. Rev. Lett. 1996, 76, 1212-1215.

15. Haucke, G.; Czerney, P.; Cebulla, F. Ber. Bunsenges. Phys. Chem. 1992, 96, 880.

16. Eaton, D. F., Luminescence Spectroscopy. In CRC Handbook of Organic Photochemistry, Scaiano, J. C., Ed. CRC Press, Inc.: Boca Raton, Florida, 2000; Vol. I, pp 231-239.

17. Meech, S. R.; Phillips, D. J. Photochem. 1983, 23, 193.

18. Gaussian 03, Revision A.1, M. J. Frisch, G. W. Trucks, H. B. Schlegel, G. E. Scuseria, M. A. Robb, J. R. Cheeseman, J. A. Montgomery, Jr., T. Vreven, K. N. Kudin, J. C. Burant, J. M. Millam, S. S. Iyengar, J. Tomasi, V. Barone, B. Mennucci, M. Cossi, G. Scalmani, N. Rega, G. A. Petersson, H. Honda, O. Kitao, H. Nakai, M. Klene, X. Li, J. E. Knox, H. P. Hratchian, J. B. Cross, C. Adamo, J. Jaramillo, R. Gomperts, R. E. Stratmann, O. Yazyev, A. J. Austin, R. Cammi, C. Pomelli, J. W. Ochterski, P. Y. Ayala, K. Morokuma, G. A. Voth, P. Salvador, J. J. Dannenberg, V. G. Zakrzewski, S. Dapprich, A. D. Daniels, M. C. Strain, O. Farkas, D. K. Malick, A. D. Rabuck, K. Raghavachari, J. B. Foresman, J. V. Ortiz, Z. Cui, A. G. Baboul, S. Clifford, J. Cioslowski, B. B. Stefanov, G. Liu, A. 18 Liashendo, P. Piskorz, I. Komaromi, R. L. Martin, D. J. Fox, T. Keith, M. A. Al-Laham, C. Y. Peng, A. Nanayakkara, 
M. Challacombe, P. M. W. Gill, B. Johnson, W. Chen, M. W. Wong, C. Gonzalez, and J. A. Pople, Gaussian, Inc., Pittsburgh PA, 2003.

19. Becke, A. D. J. Chem. Phys. 1993, 98, 5648.

20 Stephens, P. J.; Devlin, F. J.; Chabalowski, C. F.; Frisch, M. J. J. Phys. Chem. 1994, 98, 11623.

21. Cramer, C. J. Essentials of Computational Chemistry. Theories and Models. John Wiley \& Sons Ltd.: Chichester, England, 2002.

22. APEX2 Software Suite, 2.1-0; Bruker AXS: Madison, WI, 2004. 